\title{
Advanced Characterization of Nanoscale Bridge in Magnetic Tunnel Junction by 3-dimensional EDS Tomography
}

Kyuman Hwang, Junsoo Bae, Seungwoo Lee, Milim Park, Kwangho Park, Jin Choi, Juhyeon Ahn, Daeyoup Lee, Sujin Ahn, Gwan-hyeob Koh, Soonoh Park, Sangsup Jeong, Seokwoo Nam, Gitae Jeong, Hanku Cho, and Eunseung Jung

Semiconductor R\&D Center, Samsung Electronics Co., Ltd. San \#16, Banwol-Dong, Hwaseong-City, Gyeonggi-Do 445-701, Korea

Magnetic Random Access Memory (MRAM) has emerged as the leading candidate for future universal memory due to its non-volatility, excellent endurance and read/write performance [1]. The magnetic tunnel junction (MTJ) is a data storage element in MRAM and is basically composed of two ferromagnetic layers separated by the magnesium oxide $(\mathrm{MgO})$ tunnel barrier. The digital information stored in the MTJ is represented by its resistance level, thus parallel magnetic arrangement of two ferromagnetic layers leads to low resistance of MTJ but their antiparallel magnetic arrangement results in its high resistance [2]. $\mathrm{MgO}$ between two ferromagnetic layers was adopted to enlarge the resistance difference between two kinds of magnetic arrangements by tunneling current through $\mathrm{MgO}$.

The major problem in MTJ is the electrical short between two ferromagnetic due to nanoscale bridge across the $\mathrm{MgO}$ caused in the MTJ etching process, which leads to no tunnel current through $\mathrm{MgO}$ and no resistance difference between two kinds of magnetic arrangements. Even if a quite thin and narrow nanoscale bridge happened somewhere along the circumference of cylindrical shaped MTJ cell, it can result in the electrical short and failure, therefore it is very difficult to detect the nanoscale bridge by the conventional analysis of 2-dimensional TEM.

Several points of the failed MTJ cell show extremely low resistance due to electrical short in the MTJ resistance distribution, Fig.1 (a). Even a quite thin and narrow nanoscale bridge can cause the electrical short, therefore the conventional analysis by 2-dimensional TEM on the failed MTJ cannot reveal the nanoscale bridge, but shows the only clean side image of MTJ, as shown in Fig. 1 (b). The difficulties in detection of the nanoscale bridge along the MTJ circumference can get solved by the rotating 3-dimensional image or EDS tomography. The samples of the failed MTJ cell placed in TEM is tilted to plus or minus 40 degrees with the interval of 5 degrees, at which step its images were captured and analyzed. After those images were synthesized and simulated, 3-D tomography rotating laterally 360 degrees can be created, from which the nanoscale bridge happened somewhere along the circumference of the failed MTJ cell can be scanned and detected. Fig. 2 (a) is a STEM image of the failed MTJ that cannot reveal the nanoscale bridge between two ferromagnetic layers, but the STEM image in Fig. 2 (b) tilted at 20 degrees from the initial Fig. 2 (a) can reveal the nanoscale bridge at the side of MTJ. The STEM images of Fig. 2 are usually synthesized and simulated into the rotating 3-D tomography because of the contrast variation of crystalline structure with angle in TEM image. Fig. 3 (a) is the still image captured at an angle from the 3-dimensional image tomography rotating laterally, in which there is no nanoscale bridge, but the still image of Fig. 3 (b) captured at different angle shows the nanoscale bridge at the side of MTJ. The composition of nanoscale bridge across the $\mathrm{MgO}$ is analyzed to be tungsten by 3-D energy dispersive spectroscopy (EDS) tomography in Fig. 3 (c), which is originally at the top of the MTJ and redeposited at the side of MTJ in the etching process. The conventional analysis by 2-D TEM often faces its limit, so further analysis technology; 3-D EDS tomography can detect the hidden cause of failure, and extend its analysis limit. 


\section{References}

[1] W.Kim et al, IEDM Tech. Dig., 531 (2011)

[2] X.Zhu et al, IEEE Trans. Magn. 422739 (2006)
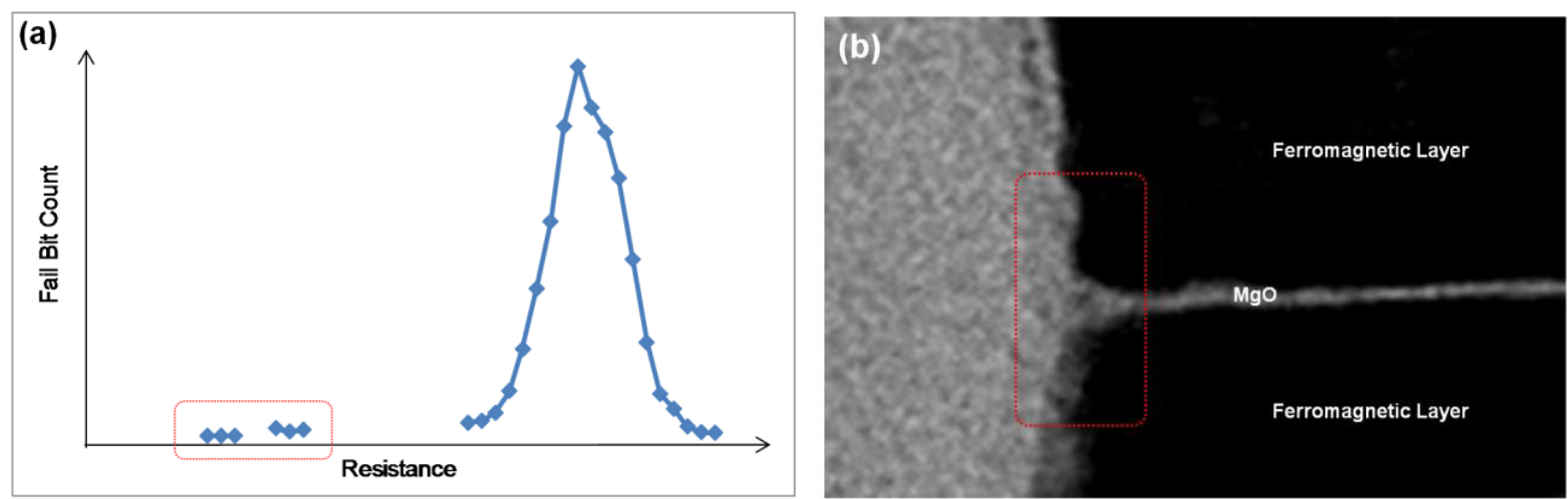

Fig. 1 (a) Resistance distribution of MTJ in the antiparallel magnetic arrangement. Several points of the failed MTJ show extremely low resistance due to electrical short. (b) 2-dimensional TEM image of the failed MTJ with electrical short cannot reveal the nanoscale bridge at the side of MTJ.
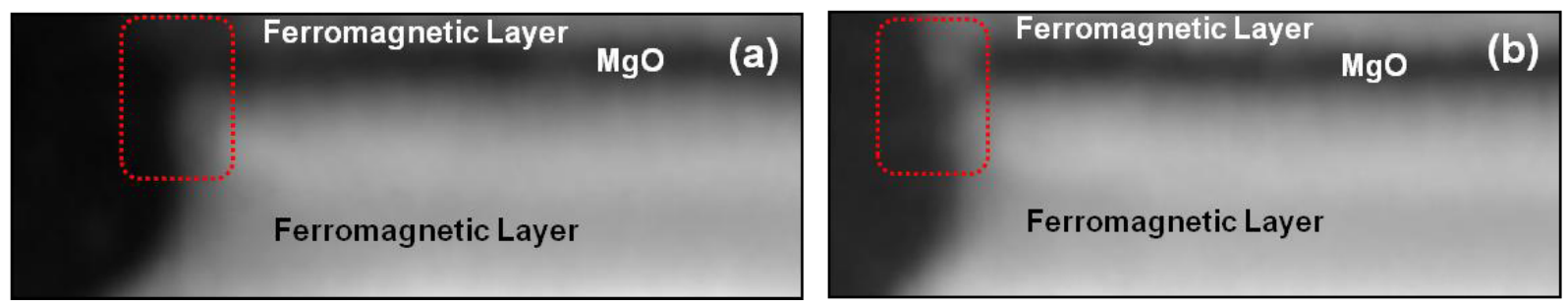

Fig. 2 (a) STEM image of the failed MTJ that cannot reveal the bridge between two ferromagnetic layers. (b) STEM image tilted at 20 degrees from Fig. 2 (a) reveal the nanoscale bridge at the side of MTJ.
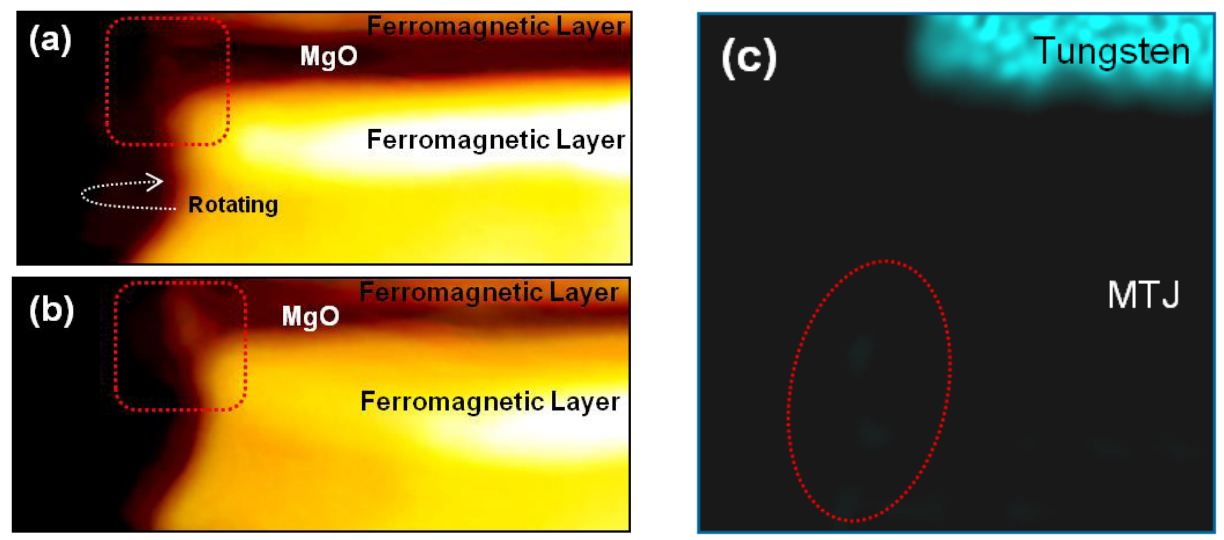

Fig. 3 (a) Still image captured at an angle from the rotating 3-dimensional tomography, in which there is no nanoscale bridge (b) Still image captured at different angle shows the nanoscale bridge at the side of MTJ (c) Still image from the rotating 3-dimensional EDS tomography reveal that the composition of nanoscale bridge is the tungsten redeposited from top of the MTJ in the etching process. 